\title{
A rare case of pituitary chromophobe carcinoma in a dog: clinical, tomographic and histopathological findings
}

\author{
M. Longo ${ }^{1,2 *}$, D. Binanti ${ }^{3}$, P.G. Zagarella ${ }^{2}$, F. Iocca $^{2}$, D. De Zani ${ }^{1}$, G. Ravasio ${ }^{1}$, M. Di Giancamillo ${ }^{1}$ and D.D. Zani ${ }^{1}$ \\ ${ }^{1}$ Dipartimento di Medicina Veterinaria (DIMEVET), Università degli Studi di Milano, Az.Polo Veterinario di Lodi, \\ Lodi, Italy \\ ${ }^{2}$ Centro Traumatologico Ortopedico Veterinario, Arenzano (GE), Italy \\ ${ }^{3} A b L a b$, Veterinary Diagnostic Laboratory, Sarzana (SP), Italy
}

\begin{abstract}
A 9 year old male mixed-breed dog was presented for progressive aggressiveness towards the owner. The neurological evaluation was consistent with a forebrain syndrome. Magnetic Resonance Imaging (MRI) of the brain revealed enlargement of the third ventricle and presence of a large spheroidal neoplasm in the sellar/parasellar region suggestive of a pituitary macroadenoma. On the owner request, the dog was euthanized. Histopathological examination revealed the presence of a pituitary chromophobe carcinoma. To the author's knowledge, pituitary carcinomas have been rarely described in dogs, especially the chromophobe subtype.
\end{abstract}

Keywords: Brain, Chromophobe carcinoma, Histology, Pituitary neoplasm, Tumour.

\section{Introduction}

Pituitary tumors are considered the most common intracranial neoplasms in dogs, making up $25 \%$ of secondary intracranial neoplasms in middle-aged and geriatric patients (O'Brien and Coates, 2010). However, in people they only account for $10-12 \%$ of all intracranial tumors (Oruçkaptan et al., 2000). The classification of these neoplasms is based on their size and in humans there is a $10 \mathrm{~mm}$ cut off that differentiates between pituitary micro and macroadenomas, with giant pituitary adenomas having a diameter of $50 \mathrm{~mm}$ (Fracassi et al., 2014). In dogs sellar/parasellar pituitary neoplasms typically refer to micro and macroadenomas and the terminology enlarged and non-enlarged has only recently been introduced, considering that even large adenomas can measure less than $10 \mathrm{~mm}$ in diameter (Fracassi et al., 2014).

Clinical signs are mainly related to the serial hormonal dysfunction that leads to Pituitary Dependent Hypercortisolism (PDH) and/or the mass effect on the adjacent brain tissues. This is more significant in the case of large neoplasms. The malignancy of a pituitary lesion is reserved in humans for pituitary tumors that show invasiveness towards the adjacent structures and even local or distant metastatic behaviour (Pollard et al., 2010; Kopczak et al., 2014). In veterinary literature there are few reports concerning pituitary adenocarcinomas, especially the chromophobe subtype (Shimada et al., 1996; Sato et al., 2001) and a recent review of 33 cases showed a $6 \%$ prevalence of adenocarcinomas, confirmed by imaging results and subsequent histopathology (Pollard et al., 2010). The present report describes clinical, tomographic, and histopathological features of a pituitary chromophobe carcinoma in a dog.

\section{Case Details}

A 9-year-old, male mixed-breed dog was referred for progressive aggressiveness towards its owner. At the clinical examination, the patient presented normothermic, polypnoic ( $>50 \mathrm{apm})$, and tachycardic (>140 bpm). Neurological evaluation revealed mental depression, normal gait, and normal postural reaction consistent with a forebrain syndrome. Due to the aggressiveness of the patient, a complete neurological examination was not performed. The diagnostic procedure included blood analysis with leukocyte formula, chest x-rays, abdominal ultrasound (with no relevant findings detected), and brain Magnetic Resonance Imaging (MRI). MRI scans were performed with a low field MRI unit $(0,2 \mathrm{~T})$ model Vet-MR ${ }^{\circledR}$ (Esaote S.p.A. Genova, Italy) and $4 \mathrm{~mm}$ thick T1-weighted pre and post paramagnetic contrast administration, T2-weighted and FLAIR sequences. At the end of the MRI exam, a total body CT scan was carried out employing a 16-slice CT unit model

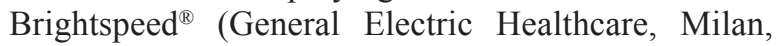
Italy), with a pitch of 1, a slice thickness of $1.25 \mathrm{~mm}$, $120 \mathrm{kV}$ and $200 \mathrm{~mA}$.

MRI revealed a moderate ventricular asymmetry, discrete left deviation of the falx cerebri, enlargement of the third ventricle and the presence of a large $(18 \times 20 \times 15 \mathrm{~mm})$ spheroidal mass in the sellar/parasellar region characterized by isointense on $\mathrm{T} 1$ weighted images (Fig. 1a,f) and increased signal on T2 weighted (Fig. 1b,e) and FLAIR (Fig. 1c). In the dorso-lateral portion of the mass, a circular lesion (6 mm diameter) 
characterized by intense and homogeneous signal hyperintensity on T2 weighted images was detected (Fig. 1e). After gadolinium-based contrast medium was administered intravenously $(0.2 \mathrm{ml} / \mathrm{kg})$, the mass showed heterogeneous intense enhancement (Fig. 1d). A pituitary enlarged adenoma/macroadenoma (invasive adenoma/adenocarcinoma) characterized by the presence of a necrotic/cystic lesion was suspected. Craniopharyngioma was also considered and included as a less likely MRI differential diagnosis. At the CT examination, no metastasis was observed in the chest and abdomen. Moreover, no adrenal hyperplasia was detected, confirming the normal blood tests and the non-secreting nature of the pituitary lesion. Due to the invasive nature of the lesion and the aggressive behavior of the patient the owner elected not to pursue further treatment. The animal was euthanized and the brain was fixed in $10 \%$ buffered formalin and submitted for histopathology examination, after written informed consent was obtained from the owner. Samples were processed routinely, embedded in paraffin wax, and sections were stained with hematoxylin and eosin. Pituitary gland was completely effaced by a wide, infiltrative, unencapsulated, not well circumscribed, densely cellular neoplasm composed of polygonal cells arranged in nests and packets supported by a fine fibrovascular stroma with numerous small hyperemic vessels (Fig. 2a). Neoplastic cells had variably distinct cell borders, intermediate $\mathrm{N} / \mathrm{C}$ ratio, moderate amount of eosinophilic cytoplasm occasionally finely granular
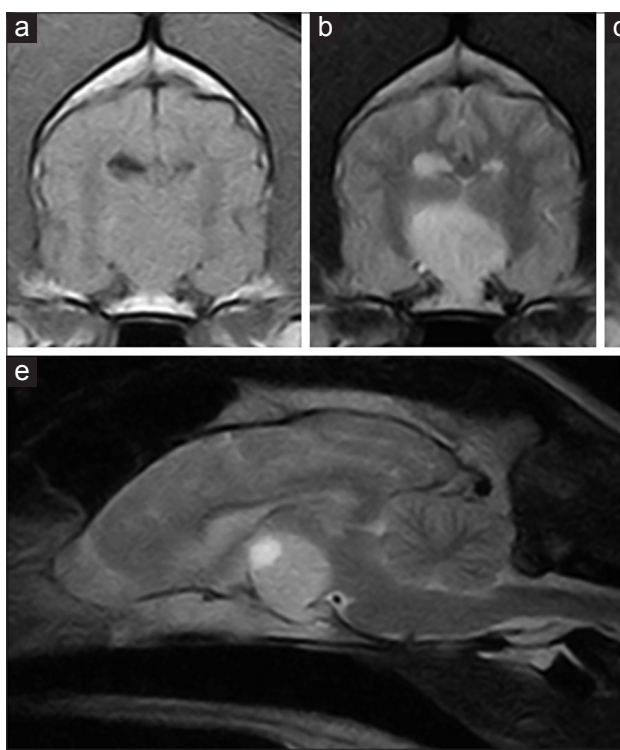
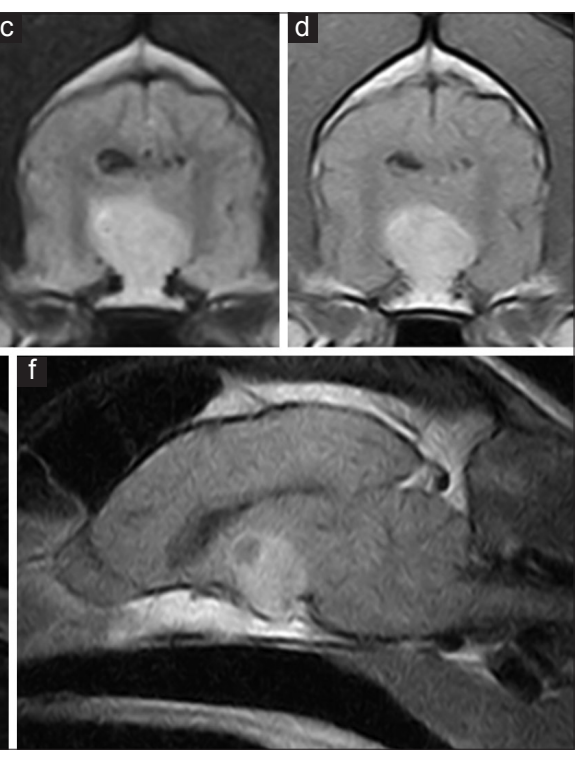

Fig. 1. Transverse (a,b,c,d) and Sagittal (e,f) MRI images of the brain showing a large spheroideal mass in the sellar/parasellar region. The mass was characterized by isointense signal on T1-weighted images (a) and high signal on T2-weighted (b and e) and FLAIR (c) sequences. A circular lesion hypointense on T1-weighted (f) and hyperintense on T2-weighted images was observed in the dorso-lateral aspect of the mass. After contrast medium administration, the mass showed a heterogeneous intense enhancement. Note the ventricular asimmetry and the moderate deviation of the falx cerebri on the transverse images of the brain $(a, b, c, d)$.

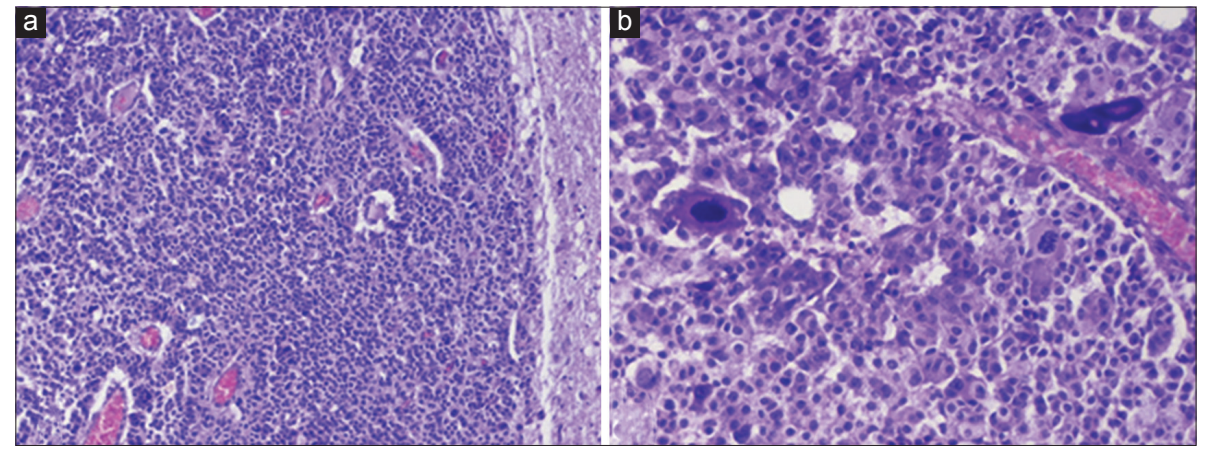

Fig. 2. Histological findings of the pituitary gland. (a) Histological examination showed a wide, infiltrative, unencapsulated, not well circumscribed, densely cellular neoplasm composed of polygonal cells arranged in nests and packets supported by a fine fibrovascular stroma with numerous small hyperemic vessels. (b) Details of the neoplastic cells with severe anisokaryosis, anisocytosis and karyomegaly. 
(Fig. 2a,b). Nuclei were round to ovalar, with one or two magenta nucleoli. Mitosis ranged from 0 to 1 per HPF ( 2 mitosis in $10 \mathrm{HPF}$ ). Severe anisokaryosis, anisocytosis and karyomegaly were evident with rare binucleated cells (Fig. 2b). The neoplasm had infiltrative growth throughout the adjacent brain parenchyma, with invasion of the third ventricle. A focal marginal residual area of pituitary parenchyma was evident. The other portion of evaluated brain was characterized by mild gliosis and satellitosis. No other neoplastic cerebral lesions were detected. Histological findings supported the diagnosis of pituitary chromophobe carcinoma.

\section{Discussion}

Pituitary carcinomas have been rarely observed in old dogs; moreover, cases of pituitary neoplasm with intense cellular pleomorphism and elevate mitotic index in absence of metastatic lesions are extremely rare. These neoplasms can cause serious functional disorders due to the destruction of the pars distalis of the neurohypophysis. Even though different therapeutical approaches have been described in literature, including trans-sphenoidal surgery and adjuvant therapies like radiotherapy (Oruçkaptan et al., 2000; Hanson et al., 2005; Fracassi et al., 2014), little evidence, concerning large pituitary tumors, of surgical outcome have been described in literature. In the present case the owner decided to euthanize the patient and histopathology revealed the infiltrative nature of the neoplasm towards the adjacent brain tissue.

Cranipharyngioma was considered as a less likely MRI differential diagnosis due to the rounded shape of the neoplasm in continuity with the hypophysis and its sellar/parasellar location peculiar for pituitary tumours. In fact craniopharyngiomas have been rarely reported in dogs (Hawkins et al., 1985; Eckersley et al., 1991) and cats (Nagata et al., 2005), with a more suprasellar and pharyngeal localization, sometimes adjacent to the dura (Hawkins et al., 1985).

Due to the absence of clinical findings compatible with hormonal imbalance or hormonal problem the neoplasm was considered a not-hormonally active tumor, and immunohistochemistry for pituitary hormones was therefore not performed.

In case of not-hormonally active neoplasm, the histological finding is the only strictly clarified method to classify the tumor. Previously a similar neoplasm was reported (Hawkins et al., 1985), based only on histological findings.

The images in the paper show severe anisokaryosis and anisocytosis typical of malignant chromophobe neoplasm. Neoplastic cells were polygonal with a small to moderate amount of eosinophilic to faintlybasophilic finely granular cytoplasm and a large, oval nucleus compatible with chromophobic cells.
In humans the distinction between invasive adenoma and pituitary adenocarcinoma is based on the finding of intracranial or systemic metastasis. It is believed that adenocarcinomas originate from malignant transformation of pre-existing adenomas after a variable latency period. In the presented case, despite the absence of systemic and intracranial metastasis, the infiltrating growth pattern and the presence of neoplastic cells that reach and surround the third ventricle, together with the intense cellular pleomorphism support the diagnosis to a rare malignant form of the neoplasm.

\section{References}

Eckersley, G.N., Geel, J.K. and Kriek, N.P. 1991. A craniopharyngioma in a seven-year-old dog. J. S. Afr. Vet. Assoc. 62, 65-67.

Fracassi, F., Mandrioli, L., Shehdula, D., Diana, A., Grinwis, G.C. and Meij, B.P. 2014. Complete Surgical Removal of a Very Enlarged Pituitary Corticotroph Adenoma in a Dog. J. Am. Anim. Hosp. Assoc. 50, 192-197.

Hanson, J.M., van't, H.M., Voorhout, G., Teske, E., Kooistra, H.S. and Meij, B.P. 2005. Efficacy of Transsphenoidal Hypophysectomy in Treatment of Dogs with Pituitary-Dependent Hyperadrenocorticism. J. Vet. Intern. Med. 19, 687-694.

Hawkins, K.L., Diters, R.W. and McGrath, J.T. 1985. Craniopharyngioma in a dog. J. Comp. Pathol. 95, 469-474.

Kopczak, A., Renner, U. and Karl Stalla, G. 2014. Advances in understanding pituitary tumors. F1000Prime Rep. 6, 5. http://doi.org/10.12703/ P6-5.

Nagata, T., Nakayama, H., Uchida, K., Uetsuka, K., Yasoshima, A., Yasunaga, S., Masuda, K., Tsujimoto, H., Kuwajima, E., Nishimura, R., Sasaki, N. and Doi, K. 2005. Two cases of feline malignant craniopharyngioma. Vet. Pathol. 42, 663-665.

O'Brien, D.P. and Coates, J.R. 2010. Brain disease. In Textbook of Veterinary Internal Medicine Expert Consult, Eds. Ettinger, S.J. and Feldman E.C. St. Louis, Missouri, USA: Elsevier Saunders, pp: 1445.

Oruçkaptan, H.H., Senmevsim, O., Ozcan, O.E. and Ozgen, T. 2000. Pituitary Adenomas: Results of 684 Surgically Treated Patients and Review of the Literature. Surg. Neurol. 53, 211-219.

Pollard, R.E., Reilly, C.M., Uerling, M.R., Wood, F.D. and Feldman, E.C. 2010. Cross-Sectional Imaging Characteristics of Pituitary Adenomas, Invasive Adenomas and Adenocarcinomas in Dogs: 33 Cases (1988 -2006). J. Vet. Intern. Med. 24, 160-165.

Sato, J., Sato, R., Kinai, M., Tomizawa, N., Osawa, T., Nakada, K., Yano, A., Goryo, M. and Naito, Y. 
2001. Pituitary Chromophobe Carcinoma with a Low Level of Serum Gonadotropin and Aspermatogenesis in a Dog. J. Vet. Med. Sci. 63, 183-185.
Shimada, A., Hara, K., Umemura, T., Kagota, K., Yamaga, Y., Ozaki, K. and Narama, I. 1996. Nonfunctional Pituitary Chromophobe Adenoma in a Calf. J. Comp. Pathol. 115, 89-93. 